\title{
Morfometría Geométrica del Borde Borial y del Collar de Huevos de Cinco Especies del Género Rhodnius Stal (Heteroptera, Reduviidae, Triatominae) ${ }^{1}$
}

\author{
Ximena Páez-Colasante ${ }^{2} \&$ Elis Aldana ${ }^{3}$
}

1. Agência de Financiamento: ULA CDCHT: C-1578-08-03-F. 2. Universidad de Los Andes, Mérida, Venezuela, Laboratorio de Entomología "Herman Lent"- Departamento de Biología, Facultad de Ciencias, e-mail: labenthl@ula.ve, 3.Universidad de Los Andes, Mérida, Venezuela, Laboratorio de Entomología "Herman Lent"- Departamento de Biología, Facultad de Ciencias, e-mail: aldana@ula.ve. Autor para correspondência.

\section{EntomoBrasilis 1(3): 57-61 (2008)}

Resumen. La taxonomía y sistemática de la subfamilia Triatominae (Heteroptera, Reduviidae) se basan principalmente en caracteres biométricos y morfológicos de las formas adultas y, en algunos casos, de las ninfas. La Morfometría Geométrica es capaz de descomponer la forma en sus componentes tamaño isométrico y conformación. En el presente trabajo se utilizó la Morfometría Geométrica como herramienta de análisis de la configuración geométrica que resulta de la unión de los extremos del borde corial y del collar (configuración borde corial-collar) de los huevos eclosionados de cinco especies del género Rhodnius Stal. Los huevos se observaron por Microscopía Electrónica de Barrido y las imágenes digitalizadas fueron analizadas morfogeométricamente. Estudiando el tamaño geométrico de la configuración borde corial-collar, se halló que no existen diferencias estadísticamente significativas entre las cinco especies de Triatominos. En cuanto a la conformación, se determinaron diferencias entre las especies al ser sometidas a un análisis discriminante. Se encontró además que la variación del tamaño contribuye a la variación en la conformación, es decir, efecto alométrico. Los resultados obtenidos indican que la conformación de la configuración geométrica "borde corial-collar" de los huevos de Rhodnius podría ser utilizada como herramienta taxonómica para este grupo.

Palabras Clave: Huevos, Análisis Generalizado de Procrustes, Morfometría Geométrica, Triatominae.

\section{Geometric Morphometrics of the Chorial Rim and the Collar of the Eggs of Five Species of the Rhodnius Genus (Heteroptera, Reduviidae, Triatominae)}

\begin{abstract}
The taxonomy and systematics of the Triatominae subfamily are based primarily on the biometric and morphological characters of the adult forms, and in some cases of the nymphs. Geometric Morphometrics are capable of separating the form in its components size and shape. In this work Geometric Morphometrics were used as a tool for the analysis of the chorial rim and the collar of hatched eggs of five species of the Rhodnius genus. Collected eggs were observed by Scanning Electron Microscopy and the digitalized images were analyzed by Generalized Procrustes Analysis. By studying the isometric size of the chorial rim and the collar, no statistical significant differences were found among the five species of Rhodnius genus. A discriminant analysis showed statistical differences among the shapes of the chorial rim and the collar, indicating that the shape is a species specific character. An allometric effect was found. The results obtained indicate that the geometric morphometrics of the chorial rim and the collar of hatched Rhodnius eggs can be used as a taxonomic tool for this group.
\end{abstract}

Key words: Eggs, Generalized Procrustes Analysis, Geometric Morphometrics, operculum, Triatominae.

$\mathbf{L}$ os Triatominos pertenecen al Orden Heteroptera, Familia Reduviidae y Subfamilia Triatominae; esta Subfamilia difiere de otras subfamilias fundamentalmente por un comportamiento estrictamente hematofágico (LENT \& WYGodzinsky 1979). La importancia de esta subfamilia se debe a la capacidad vectora que algunas especies poseen para la transmisión de la enfermedad de Chagas, la cual es una zoonosis producida por un protozoario flagelado conocido como Trypanosoma cruzi (Chagas). Sólo aquellas especies con adaptaciones para vivir en el domicilio humano son consideradas vectores epidemiológicamente importantes (ZELEDón 1983).

Según Galvão et al. (2003) el género Rhodnius Stal agrupa a 16 especies que se distribuyen a lo largo de toda Sudamérica y América Central. El género Rhodnius se ha caracterizado basándose en la morfología externa de ninfas, adultos y de la genitalia externa del macho, en el comportamiento alimenticio y reproductivo y en la biometría y morfología de los huevos (LENT 1948; LENT \& JURBERG 1969; LENT \& WYGODZINSKY 1979; Barata et al. 1980; Barata 1981; Barata 1998; Aldana et al. 2000; Aldana et al. 2001).

Entre los primeros trabajos que se conocen acerca de los huevos de los Triatominos, está el realizado con la técnica de la Microscopia Óptica (MO) por BricEÑo-IRAGORRY (1934) el cual describe que los huevos de Rhodnius prolixus Stal poseen células poligonales y que son impresos por células epiteliales de las glándulas ováricas; la geometría de estos huevos fue corroborada por GALLIARD (1935) y BARTH \& Muth (1958).

LENT \& Wygodzinsky (1979) señalaron que los huevos del género Rhodnius se diferencian de otros géneros por presentar constricción en el cuello y reticulaciones del corion de forma cóncava y perforadas en la región media. BARATA (1981) y BARATA (1998) estudiaron las características macroscópicas y exocoriales de los huevos del género Rhodnius y propone claves dicotómicas para la identificación de las especies. La región cefálica del huevo del género Rhodnius se caracteriza por una constricción (el cuello) entre el borde corial y el collar (LENT \& WYGODZINSKY 1979); tanto el cuello como el collar pueden ser más o menos conspicuos según la especie (BARATA 1998).

Por otra parte, la superficie de los huevos de Rhodnius comprende células hexagonales compuestas a su vez de una zona periférica estrecha, plana, con numerosas granulaciones irregulares de distribución acentuada hacia los bordes y de una cavidad central en forma de embudo que penetra profundamente en el corion, el cual se continúa hasta el fondo con un pequeño tubo terminal curvado cuya extremidad es cerrada (Barata et al. 1998).

Zamora et al. (2000) observaron que la distribución de las formas poligonales en la superficie del corion de los huevos de $R$. prolixus varía según se trate de las regiones caudal y cefálica o de la región media del huevo, y que dicha variación es debida 
a una necesidad estructural, ya que es lo que permitiría que la superficie del huevo se cierre. CHAves \& ÁñEz (2003) estudiaron mediante morfometría tradicional la geometría del exocorion de $R$. prolixus y encontraron que la presencia de células poligonales de geometría similar no varían durante el desarrollo embrionario en las diferentes zonas del huevo.

Mediante Morfometría Geométrica (MG) se han analizado las alas y cabezas de varias especies del género Rhodnius (MATIAS et al. 2001; VILLEGAS et al. 2002; JARAMILlO et al. 2002; MoJica et al. 2005; Feliciangeli et al. 2007), pero no se ha empleado este enfoque analítico en los huevos de este género ni de ningún otro grupo de Triatominos.

En el presente estudio se amplía el conocimiento de la morfología del huevo mediante el Análisis Generalizado de Procrustes de cinco especies del género Rhodnius, R. prolixus, Rhodnius robustus Larrousse, Rhodnius nasutus Stal, Rhodnius neivai Lent y Rhodnius neglectus Lent, con el fin de contribuir con información de interés a la taxonomía del género.

\section{Material y Métodos}

Los huevos estudiados provenían de 15 parejas por especie mantenidas en la colonia del Laboratorio de Entomología "Herman Lent" de la Facultad de Ciencias de la Universidad de Los Andes en Mérida, Venezuela, mantenidas a una temperatura de $26-28^{\circ} \mathrm{C}$ y a una humedad relativa de $40-50 \%$. Las parejas se alimentaron tres veces hasta repleción sobre gallina y los huevos se recolectaron luego de la eclosión.

Se colocaron 40 huevos por especie en un soporte metálico sobre cinta doble adhesiva. Los huevos se colocaron con el achatamiento lateral de la barriga, característico de los huevos del género Rhodnius, en contacto con la cinta adhesiva. Los huevos se recubrieron con plata y luego se observaron a un aumento de $150 X$ por MEB en un microscopio Hitachi S 2500 del Laboratorio de Análisis Químico y Estructural de Materiales (L.A.QU.E.M.) del Grupo de Física Aplicada del Departamento de Física de la Facultad de Ciencias de la Universidad de Los Andes.

Para el análisis se ubicaron seis Puntos Anatómicos de Referencia (PAR) o landmarks (Figura 1), cuatro en el en el borde corial y dos en el collar, con lo cual se obtuvo una configuración geométrica que en adelante llamaremos "configuración borde corial-collar”. El criterio para escoger los seis PAR fue que no se identificó simetría bilateral de la configuración borde corialcollar en todas las especies como se observa en la Figura 1.
Luego se digitalizaron dichos PAR utilizando el programa tpsDig versión 1.18 (Rohlf 2006). Posteriormente mediante el programa MOG versión 0.71 (DuJARDIN 2005) se removió el tamaño isométrico y se crearon las variables de conformación mediante el Análisis Generalizado de Procustes (AGP). Se utilizaron las variables de conformación obtenidas mediante el AGP para comparar los huevos de las diferentes especies. Además, se realizó un análisis multivariado de varianza (MANOVA) para evaluar la igualdad de las conformaciones medias. El análisis estadístico del tamaño y de la conformación se llevó a cabo con el programa Past versión 1.64 (HAMmer et al. 2001). Por último, se reconocieron los cambios de conformación que resultaron de los cambios de tamaño, es decir, la alometría, mediante el programa Padwin versión 82 (DUJARDIN 2006). Los efectos alométricos se examinaron con este mismo programa mediante un análisis de regresión lineal simple, donde las variables de conformación actuaron como variables dependientes (el primer factor canónico generado por el análisis discriminante) y el tamaño y la especie como variables independientes. Para el análisis del tamaño se empleó el estadístico no paramétrico de Kruskal-Wallis con corrección de Bonferroni. Se tomó como valor de “p” o,05.

\section{Resultados}

Tamaño isométrico. En la Figura 2 se muestran los tamaños centroides de la configuración borde corial-collar de los huevos correspondientes a las cinco especies analizadas. El estadístico no paramétrico de Kruskal-Wallis no reveló diferencias estadísticamente significativas entre las especies ( $p>0,05)$.

Conformación. En la Figura 3 se muestra la distribución de los individuos según las variables de conformación de la configuración borde corial-collar de los huevos correspondientes a las cinco especies analizadas. Mediante el estadístico de Wilk's lambda se encontraron diferencias estadísticamente significativas entre las conformaciones de las especies analizadas ( $p<0,05)$. En el análisis se obtuvieron cuatro factores discriminantes, siendo la contribución porcentual de cada uno de $73 \%$, $21 \%$, 5\% y $1 \%$. La gráfica muestra a las especies analizadas agrupadas mediante elipses de confianza de 95\%. Mediante la prueba de reclasificación que utiliza el método del "jacknife" del programa PADwin, se encontró que tal validación identificó correctamente al 97\% de $R$. prolixus, al $92 \%$ de $R$. robustus, al $57 \%$ de R. nasutus, al $77 \%$ de R. neivai y al $87 \%$ de $R$. neglectus.

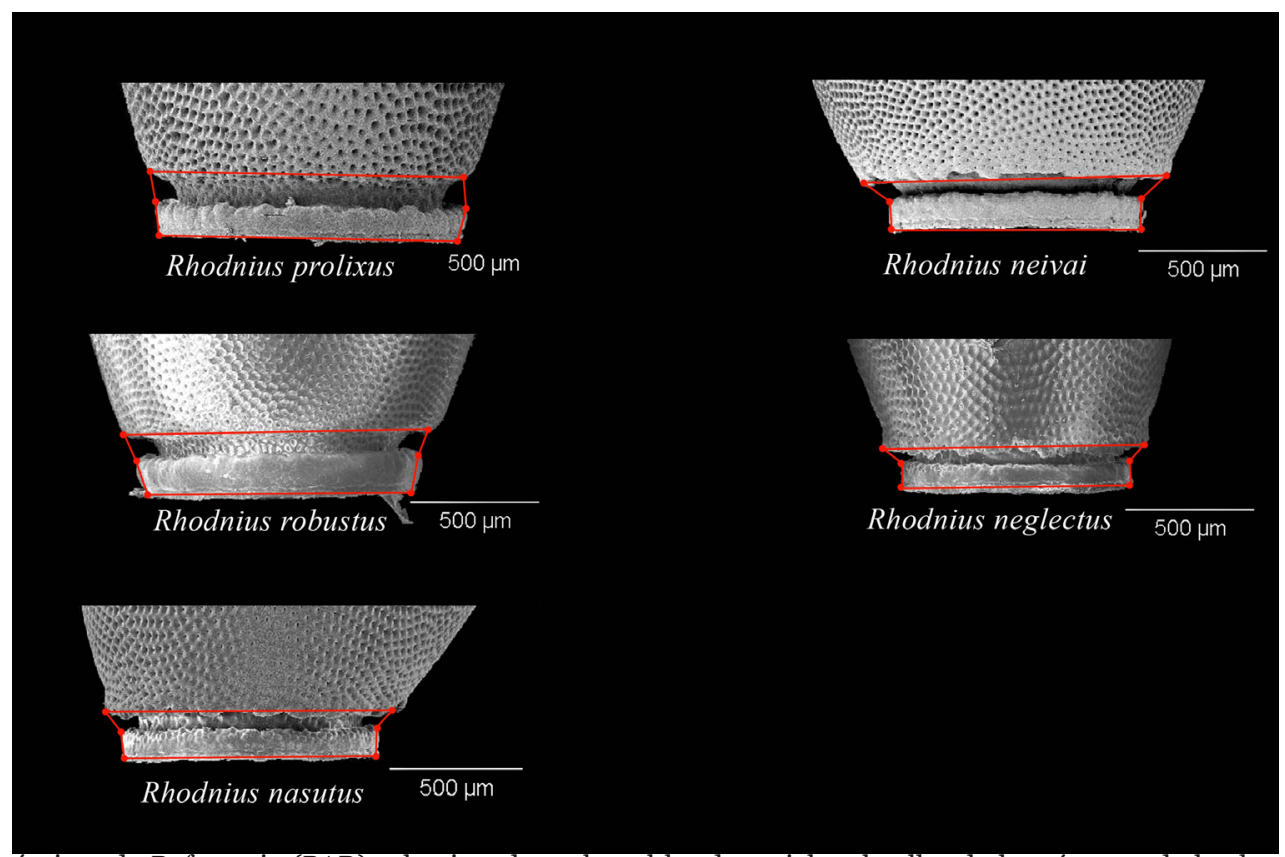

Figura 1. Puntos Anatómicos de Referencia (PAR) seleccionados sobre el borde corial y el collar de las cáscaras de los huevos de las especies $R$. prolixus, $R$. robustus, $R$. nasutus, $R$. neivai y $R$. neglectus. La unión de los PAR genera la configuración geométrica borde corial-collar analizada en el presente trabajo. 


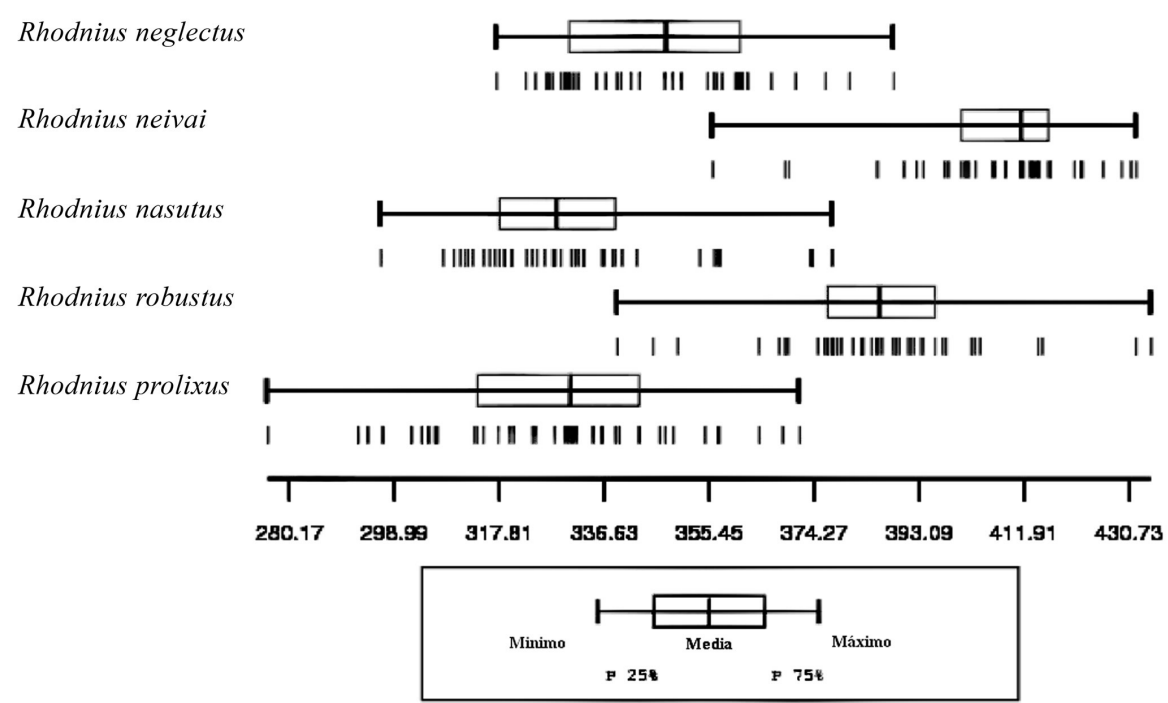

Figura 2. Variación del tamaño de la configuración geométrica borde corial-collar del huevo de $R$. prolixus, $R$. robustus, $R$. nasutus, $R$. neivai y $R$. neglectus.

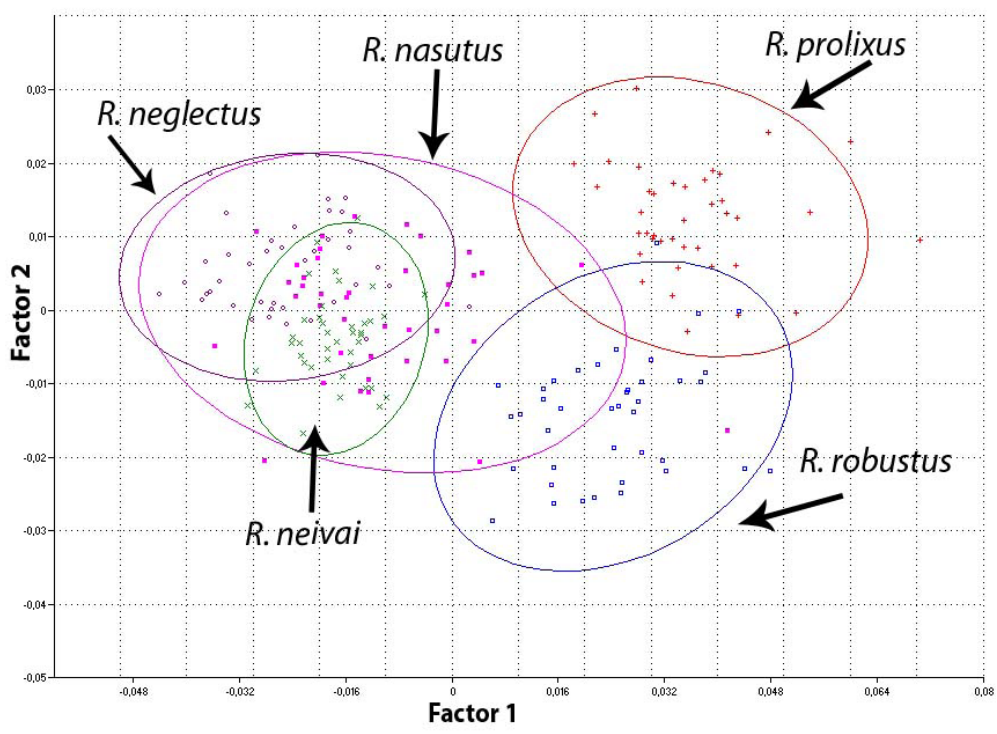

Figura 3. Diagrama factorial en las variables de conformación que se observan al hacer el análisis discriminante de la configuración geométrica borde corial-collar del huevo de $R$. prolixus, $R$. robustus, $R$. nasutus, $R$. neivai y $R$. neglectus, donde los individuos de cada especie se proyectan sobre los dos primeros factores canónicos, los cuales enmarcan el espacio multivariado de la conformación.

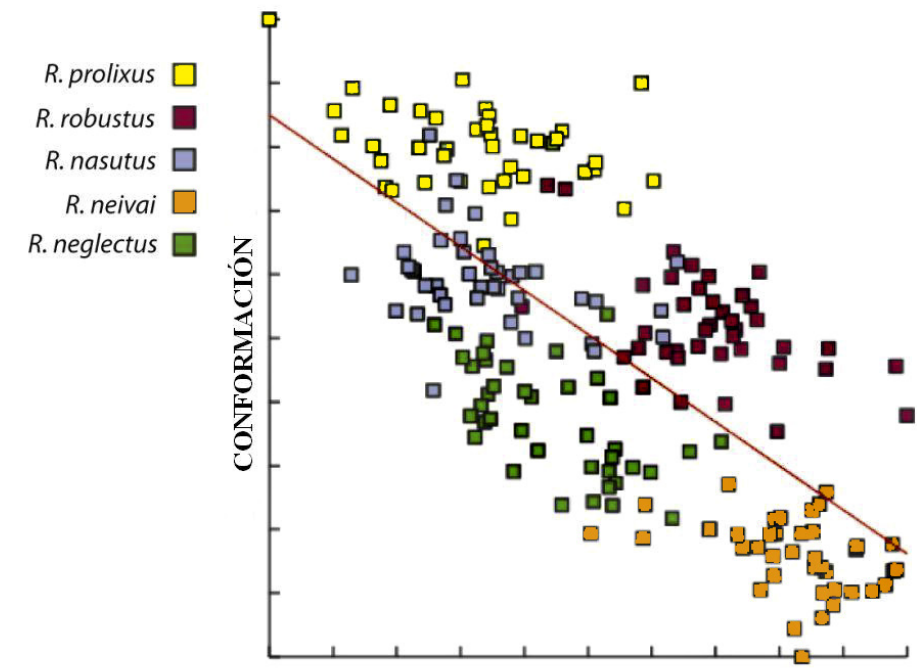

TAMAÑO

Figura 4. Efecto Alométrico: efecto del tamaño de la configuración geométrica borde corial-collar del huevo de R. prolixus, $R$. robustus, $R$. nasutus, $R$. neivai y $R$. neglectus, sobre la conformación. 
Efecto alométrico. Mediante un análisis de regresión lineal, con el tamaño como variable independiente y la conformación como variable dependiente, se muestra en la Figura 4 el efecto del tamaño sobre las variables de conformación. Mediante este análisis se observó que el tamaño contribuye en el $48 \%$ de la variación de las variables de la conformación.

\section{Discusión}

Las especies del género Rhodnius han sido caracterizadas mediante el análisis morfológico y el comportamiento de ninfas y adultos (LENT 1948; LENT \& JURBERG 1969; LENT \& WYGODZINSKY 1979; Aldana et al. 2000; Aldana et al. 2001; Sant'Anna et al. 2001) y mediante el análisis morfológico de los huevos por medio de morfometría tradicional y MEB (BARATA et al. 1980; BARATA 1981; Barata 1998; Cháves \& ÁÑEZ 2003).

El AGP ha sido empleado en el análisis de alas y cabezas del género Rhodnius (Matias et al. 2001; Villegas et al. 2002; JARAMIllo et al. 2002; MoJiCa et al. 2005; FELICIANGELI et al. 2007), pero no ha sido aplicado en huevos ni de Triatominos ni de ningún otro grupo de insectos, debido quizá a la ausencia en los huevos de estructuras anatómicas que permitan la localización de PAR tanto de tipo 1 como de tipo 2 según las categorías de Bookstein (1991). Sin embargo, dado que los huevos de las especies del género Rhodnius invariablemente poseen una región cefálica compleja y estructurada anatómicamente (BEAMENT 1946; BARATA 1981; BARATA 1998), es posible la localización de PAR en el borde corial y en el collar.

En el presente trabajo, se analizó mediante AGP la configuración geométrica que resulta de la unión de los extremos del borde corial y del collar encontrándose que no hay diferencias estadísticamente significativas en el tamaño pero sí en la conformación, con lo cual se amplía el conocimiento de caracteres que discriminan entre especies del género Rhodnius.

Dado que se conoce bien que la región cefálica y sus estructuras asociadas (opérculo, borde corial, cuello, gotera espermática, micropilis y aeropilis) están comprometidas en la viabilidad del huevo de $R$. prolixus y de los insectos en general (BEAMENT 1946), las diferencias de conformación de la configuración borde corial-collar entre las especies analizadas en el presente trabajo podrían explicar, al menos parcialmente, las diferencias entre las características bionómicas y funcionales relativas al huevo descritas por CANALE et al. (1998) en algunas especies del género Rhodnius. El análisis mediante AGP de este trabajo contribuye además con información que confirma la distinción entre las especies $R$. prolixus y $R$. robustus, para las cuales HARRY et al. (1992) propusieron una revisión taxonómica dada la coincidencia en el patrón isoenzimático.

Por otra parte en este trabajo se encontró que la variación del tamaño de la configuración borde corial-collar entre las especies analizadas contribuye a la variación de su conformación, es decir, se encontró un efecto alométrico en esta configuración geométrica. La relación alométrica con el tamaño generalmente es consecuencia de adaptaciones (Futuyma 1998), es decir, de funciones que han surgido y/o mantenido por selección natural. La configuración borde corial-collar analizada en este trabajo fue realizada sobre huevos que han sido mantenidos en un insectario en las mismas condiciones estables de humedad y temperatura, por lo que la alometría encontrada en esta configuración revela algunas de las diferencias genéticas entre las especies analizadas. Estas diferencias están comprometidas en la funcionalidad diferencial de la región cefálica del huevo.

CARCAvallo et al. (2000) proponen hipótesis filogenéticas sobre las relaciones ente las especies del género Rhodnius, mediante el agrupamiento en complejos de especies, incluyendo en el complejo prolixus a las especies $R$. prolixus, $R$. robustus, $R$. neglectus, $R$. nasutus y a $R$. domesticus, y no consideran a $R$. neivai relacionada filogenéticamente a ninguna de las especies conocidas del género Rhodnius. Pese a que en el presente trabajo no se pretende analizar filogenéticamente a estas especies mediante el AGP de la configuración borde corialcollar, cabe destacar que en cuanto a este carácter $R$. neivai no se distingue por el tamaño y se solapa en la conformación con $R$. neglectus y $R$. nasutus. Por otra parte, el hecho que $R$. nasutus haya presentado el porcentaje más bajo de reclasificación, podría interpretarse que en cuanto al carácter configuración borde corial-collar, esta especie no está tan bien naturalmente definida como el resto de las especies analizadas.

Sin embargo, no podemos inferir relaciones filogenéticas a partir de estos resultados sin antes establecer el criterio y la validez de la configuración borde corial-collar para el análisis filogenético en todas las especies del género Rhodnius, lo cual escapa al objetivo del presente trabajo.

Finalmente, se sugiere el análisis de la configuración borde corial-collar mediante el AGP de los huevos de otras especies del género Rhodnius, con el fin de verificar el carácter especie-específico de las diferencias en la conformación de dicha configuración.

\section{Agradecimientos}

A J. Fernández, Andrés Mora, E. Lizano, A. Avendaño, A. Rojas, W. Quiñones y CDCHT: C-1578-08-03-F.

\section{REFERENCIAS}

Aldana E., E. Lizano, F. Contreras, A. Valderrama \& D. Viera, 2000. Estudio morfológico de estadios ninfales de varias especies del género Rhodnius (Hemiptera Reduviidae). Caldasia, 22(2):347-351.

Aldana E., E. Lizano, O. Rodríguez \& A. Valderrama, 2001. Alimentación y defecación en triatominos del género Rhodnius (Hemiptera, Reduviidae) alimentados con sangre humana. Revista de Biología Tropical, 49(2):693-696.

Barata, J., J. Santos \& C. Leite, 1980. Aspectos morfológicos de ovos de Triatominae I. Mensuração de dez espécies do género Rhodnius Stal, 1859 (Hemiptera: Reduviidae). Revista Brasileira de Entomología, 24(3/4):197-214.

Barata, J. 1981. Aspectos morfológicos de ovos de Triatominae II. Características macroscópicas e exocoriais de dez espécies do género Rhodnius Stal, 1859 (Hemiptera: Reduviidae). Revista de Saúde Pública, 15:490-542.

Barata, J., 1998. Estructuras macroscópicas e exocoriais de ovos de Triatominae (Hemiptera, Reduviidae), p. 409- 448. En: Carcavallo R.U., I. Galíndez Girón, J. Jurberg \& H. Lent (Eds). Atlas dos vetores da Doença de Chagas nas Américas. Rio de Janeiro, Editora Fiocruz, 1217p.

Barth, R. \& H. Muth, 1958. Estudos anatômicos e histológicos sobre a subfamília Triatominae (Heteroptera, Reduviidae) VIII parte: Observações sobre a superfície dos ovos das espécies mais importantes. Memórias do Instituto Oswaldo Cruz, 56(1): 197-208.

Beament, J. W. L., 1946. The Formation and structure of the chorion of the egg in an hemipteran, Rhodnius prolixus. Quarterly Journal of Microscopical Science, s2-87 (348):393439.

Bookstein, F.L. 1991. Morphometric tools for landmark data: geometry and biology. Cambridge, Cambridge University Press, 433p.

Briceño-Iragorry, L. 1934. Contribución al estudio morfológico de los huevos de algunos reduvideos. Boletín de la Sociedad Venezolana de Ciencias Naturales, 2:397-400.

Canale, D., J. Jurberg, R. Carcavallo, C. Galvao, I. Galíndez Jirón, C. Segura, D. Rocha \& A. Martínez, 1999. Bionomics of some species, p. 839-890. En: Carcavallo R.U., I. Galíndez Girón, J. Jurberg \& H. Lent (Eds). Atlas dos vetores da Doença de Chagas nas Américas. 1217 p. Rio de Janeiro, Editora Fiocruz, 1217p.

Carcavallo, R.U., J. Jurberg, H. Lent, F. Noireau \& C. Galvão, 
2000. Phylogeny of the Triatominae(Hemiptera: Reduviidae). Proposals for Taxonomic Arrangements. Entomologia y Vectores, 7(1):1-99.

Chaves, L.F. \& N. Añez, 2003. Geometría de las células del exocorión de huevos de Rhodnius prolixus Stal, 1859 (Heteroptera: Reduviidae). Entomotropica, 18(1):1-5.

Dujardin, J.P. 2005. MOG (Morfometria Geométrica) versión 0.71. Institut de Recherches pour le Développement (IRD), Montpellier-France. Disponible en: $<$ http://life.bio.sunysb.edu/morph/index.html $>$.

Dujardin, J.P. 2006. PAD versión 82. Institut de Recherches pour le Développement (IRD), Montpellier-France. Disponible en: $<$ http://www.mpl.ird.fr/morphometrics $>$.

Feliciangeli, M.D., M. Sanchez, R. Marrero, C.Y. Davies \& P.J. Dujardin, 2007. Morphometric evidence for a possible role of Rhodnius prolixus from palm trees in house re-infestation in the State of Barinas (Venezuela). Acta Tropica, 101(2): 169177.

Futuyma, D.J. 1998. Evolutionary Biology. Sunderland, Sinauer Associates, Inc., 763p.

Galvão, C., R. Carcavallo, D. Rocha, J. Jurberg, 2003. A checklist of the current valid species of the subfamily Triatominae Jeannel, 1919 (Hemiptera, Reduviidae) and their geographical distribution, with nomenclatural and taxonomic notes. Zootaxa, 202: 1-36

Galliard, H. 1935. Recherches sur les Réduvides Hématophages Rhodnius et Triatoma. V Morphologie de l'oeuf des Triatomines. Annales de Parasitologie Humaine et Comparee, 13(6):511-527.

Hammer, Ø., D.A.T. Harper \& P. D Ryan, 2001. PAST: Paleontological Statistics Software Package for Education and Data Analysis. Palaeontologia Electronica. 4(1): 9pp. Disponible en: $<$ http://palaeo-electronica.org/2001_1/past/issue1_01.htm>.

Harry, M., I. Galíndez \& M. Cariou, 1992. Isozyme variability and differentiation between Rhodnius prolixus, $R$. robustus and $R$. pictipes, vectors of Chagas disease in Venezuela. Medical and Veterinary Entomology, 6:37-43.

Jaramillo, N., L.A. Calle, H. Caro - Riaño, J. Calle \& J. OrtegaBarría, 2002. Diferencias morfométricas asociadas a la distribución de Rhodnius pallescens provenientes de siete localidades de Colombia y Panamá. Taller Técnico de Estudio sobre Rhodnius pallescens, su Vigilancia y Control. Organización Panamericana de la Salud. 15-23.

Como citar este artigo:

Páez-Colasante X. \& E. Aldana, 2008. Morfometría Geométrica del Borde Corial y del Collar de Huevos de Cinco Especies del Género Rhodnius Stal (Heteroptera, Reduviidae, Triatominae). EntomoBrasilis, 1(3): 57-61. www.periodico.ebras.bio.br/ojs
Lent, H., 1948. O gênero Rhodnius Stal, 1859 (Hemiptera, Reduviidae). Revista Brasileira de Biología, 8(3):297-339.

Lent, H. \& J. Jurberg, 1969. O gênero Rhodnius Stal, 1959, com um estudo sobre a genitália das espécies (Hemiptera, Reduviidae, Triatominae). Revista Brasileira de Biologia, 29(4):487-560.

Lent, H. \& P. Wygodzinsky, 1979. Revision of the Triatomines (Hemiptera, Reduviidae) and their significance as vector of Chagas Disease. Bulletin of American Museum of Natural History, 163(3):123-520.

Matias, A., J.X. De la Riva, M. Torrez \& J.P. Dujardin, 2001. Rhodnius robustus in Bolivia Identified by its Wings. Memorias do Instituto Oswaldo Cruz, Rio de Janeiro. 96(7): 947-950.

Mojica, M.T., G. Aguilera, N. Pinto \& F. Guhl, 2005. Morfometría geométrica aplicada a Rhodnius prolixus, Triatoma dimidiata y T. maculata, p.315-338. En: Guhl, F. (Ed.). Primer taller internacional sobre control de la enfermedad de Chagas. Curso de diagnóstico, manejo y tratamiento de la enfermedad de Chagas. VI reunión de la iniciativa andina para el control de la enfermedad de Chagas. Bogotá D.C., Corcas Editores, p. 435 .

Rohlf, F. J., 2006. TPSdig, Version 1.18. Departamento of ecology and evolution state University of New York Stony Brook, NY. Disponible en: <http://life.bio.sunysb.edu/morph/index.html $>$.

Sant'Anna, M.R.V., L. Diotaiuti, A.Gontijo, N. Gontijo \& M.H. Pereira, 2001. Feeding behaviour of morphologically similar Rhodnius species: influence of mechanical characteristics and salivary function. Journal of Insect Physiology, 1459-1465.

Villegas, J., Feliciangeli M.D \& J.P. Dujardin, 2002. Wing shape divergence between Rhodnius prolixus from Cojedes (Venezuela) and Rhodnius robustus from Mérida (Venezuela). Infection, Genetics and Evolution, 2:121-128.

Zamora, E., L.F. Chaves, E. Aldana, E. Lizano, E. Valiente, 2000. Estudio anatómico del tubo ovárico de Rhodnius prolixus utilizando el microscopio electrónico de barrido (MEB). Acta Microscópica, 1:132-133.

Zeledón, R., 1983. Vectores de la enfermedad de Chagas y sus características ecofisiológicas. Interciencia, 8(6):384-395.

Recebido em: 14/08/2008

Aceito em: 14/10/2008

$* * * * * * * * * * * * *$
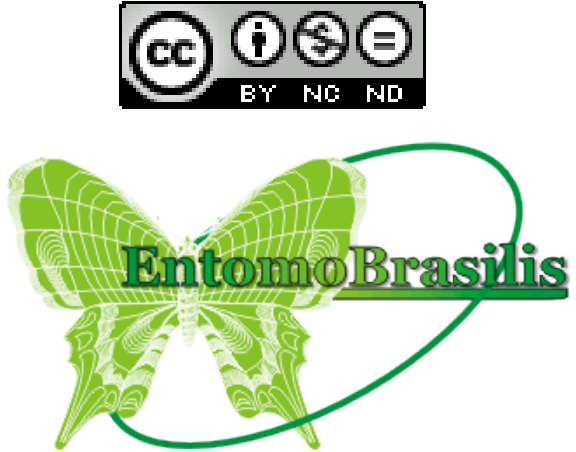


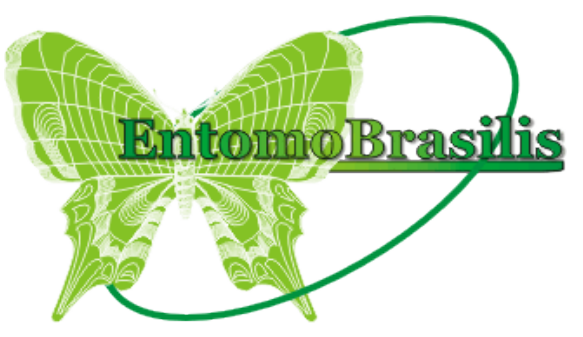

